\title{
Reducing the Risk of Breast Cancer Recurrence: an Evaluation of the Effects and Mechanisms of Diet and Exercise
}

\author{
Christina M. Dieli-Conwright $^{1} \cdot$ Kyuwan Lee $^{1} \cdot$ Jacqueline L. Kiwata $^{1}$
}

Published online: 27 June 2016

(C) Springer Science+Business Media New York 2016

\begin{abstract}
With recent medical advances in diagnosis and treatment, the increasing numbers of long-term survivors of breast cancer is considerable and has resulted in the expansion of scientific research to include examination of lifestyle modifications as means of prevention of recurrence, new breast cancer events, and mortality. The objective of this report is to review randomized controlled trials (RCTs) including diet and/or exercise interventions on breast cancer recurrence in women with a history of breast cancer as well as pertinent recent epidemiologic evidence. Implicated biologic mechanisms are discussed to elucidate the impact of diet and exercise on disease recurrence.
\end{abstract}

Keywords Breast cancer · Diet · Nutrition · Exercise · Physical activity $\cdot$ Recurrence $\cdot$ Obesity

\section{Introduction}

Given the recent advances in the diagnosis and treatment of breast cancer, the number of breast cancer survivors is steadily growing, with the 5-year relative survival rate of $91 \%$ and

This article is part of the Topical Collection on Clinical Trials

Christina M. Dieli-Conwright

cdieli@usc.edu

Kyuwan Lee

kyuwanle@usc.edu

Jacqueline L. Kiwata

kiwata@usc.edu

1 Division of Biokinesiology and Physical Therapy, University of Southern California, 1540 E. Alcazar St., CHP-155, Los Angeles, CA 90033, USA more patients living longer past diagnosis [1]. Yet, with this greater survival, the long-term effects of breast cancer and treatment-related side effects have become increasingly evident. Breast cancer survivors are at higher risk for weight gain following diagnosis regardless of menopausal status [2], and given that obesity is a risk factor for a number of adverse outcomes, including comorbid disease, recurrence or overall mortality, research has expanded to include investigations into lifestyle modifications directed towards healthy weight management. The lifestyle modifications aimed at preventing recurrence, typically defined as a relapse event at a local, regional, or distal site, have consisted of a healthful diet, nutritional supplements, regular exercise, or some combination of these components.

Though numerous reviews have been conducted on the relationship between lifestyle factors and cancer recurrence [3-6], not all have been specific to breast cancer, focused primarily on evidence from randomized controlled trials (RCTs), or discussed the physiological mechanisms underpinning lifestyle modifications in relation to breast cancer recurrence. Therefore, the purpose of this review is to summarize evidence from recent lifestyle interventions examining the role of diet, exercise, or multicomponent approaches on recurrence and mortality outcomes in breast cancer survivors. As such, this review is organized into three sections: (1) diet interventions; (2) exercise interventions; and (3) multicomponent approaches involving diet combined with exercise. Based on this body of knowledge, reasonable recommendations on diet and exercise that aim to reduce the risk of recurrence and mortality in breast cancer survivors are provided.

\section{Diet and Risk of Breast Cancer Recurrence}

Considerable evidence exists for the role of healthy eating in breast cancer prevention, while the body of support for the 
effects of diet on the risk of breast cancer recurrence is rapidly growing [3]. This section will focus on diet-only interventions rather than multicomponent approaches involving both diet and exercise, which are discussed together in a later section. Data from randomized controlled trials (RCTs) involving women with breast cancer are presented in regards to two primary dietary patterns - (1) a low-fat diet and (2) a lowfat, high fruit, and vegetable diet — and the effects of soy and vitamin D intake are also discussed.

\section{Low-Fat Diet and Risk of Breast Cancer Recurrence}

Data from large observational studies have suggested an association between higher intake of dietary fat and greater risk of breast cancer recurrence, particularly in post-menopausal women [5], motivating investigations to examine the effect of low-fat dietary interventions on breast cancer recurrence. The Women's Intervention Nutrition Study (WINS; $n=2437$ ) was the first of two large, multicenter RCTs employing a lowfat dietary intervention to promote relapse-free survival in women with early-stage breast cancer [7]. The second largescale RCT, the Women's Healthy Eating and Living study (WHEL; $n=3088$ ), incorporated a high intake of fruits and vegetables in addition to a low-fat diet [8]; hence, it will be discussed in the subsequent section. In WINS, women aged 48-79, who had completed conventional primary treatment for early-stage breast cancer including surgery, radiotherapy, tamoxifen for estrogen receptor (ER)-positive tumors, and chemotherapy for ER-negative tumors, were recruited within 1 year of diagnosis. Forty percent $(n=975)$ were randomized to the low-fat dietary intervention, where participants reduced the percentage of calories from fat to $15 \%$. Control subjects $(n=1462)$ received only written dietary guidelines. After a median of 60 months, only $9.8 \%$ of women in the diet group had reported a recurrence event compared to $12.4 \%$ of women in the control group, with a hazard ratio for relapse-free survival, defined as recurrence at any site, of 0.76 (95\% CI, 0.60 to $0.98 ; p=.034$ ). As statistically significant weight loss was observed in the intervention group compared to the control group $(p=.005)$, the dietary intervention was purported to lower the risk of recurrence through reductions in body weight. However, no statistical significance was found for an intervention effect on overall survival ( $\mathrm{HR}=0.89 ; 95 \%$ CI, 0.65 to $1.21 ; p=.56)$, although a follow-up analysis reported a significant overall survival benefit in women with ER and progesterone receptor (PR) disease (7.5 vs. $18.1 \%$ cumulative mortality, $\mathrm{RR} 0.41, p=.003, n=362$ ) [9].

\section{Low-Fat Diet and Risk of Breast Cancer Recurrence: Plausible Mechanisms}

The physiological basis for the relationship between a high-fat diet and breast cancer recurrence has been attributed to higher body adiposity and the modulatory effects of dietary fats on eicosanoid synthesis [10-12]. Higher adiposity is associated with adverse levels of insulin-like growth factor 1 (IGF-1), inflammatory markers and sex hormones, all of which may act to promote tumor development and growth [10]. The role of IGF-1 and its receptor IGF-1R in the progression of breast tumors has been demonstrated through multiple breast cancer models (reviewed in Belardi et al. [13•]). Though activation of IGF-1R alone is not an oncogenic event, survival of already differentiated cells is dependent on IGF-1/IGF-1R signaling, suggesting that the IGF-1 axis is an important factor in tumor progression [14]. Indeed, high expression of activated IGF-1R is related to poorer survival in breast cancer patients [15]. However, evidence is lacking on the direct relationship between circulating IGF-1 as a result of dietary intake and its effect on IGF-1 axis signaling and tumor progression.

On the other hand, more evidence is available to support the role of adiposity in the breast cancer progression. The accumulation of adipose tissue, specifically visceral fat, increases circulating levels of pro-inflammatory cytokines, including interleukin-6 (IL-6) and transforming growth factor beta (TGF- $\beta$ ), and the pro-inflammatory adipokine, leptin, which are secreted from the adipose tissue itself $[16,17]$. Leptin in particular has been shown to promote tumor cell growth and metastases through activation of numerous signaling pathways [16]. In addition, the resulting state of chronic inflammation from the systemic induction of proinflammatory cytokines has been shown to contribute to tumor angiogenesis and change to a metastatic phenotype [17-19]. For example, both IL-6 [19] and TGF- $\beta$ [17] induce epithelial-to-mesenchymal transition, a correlate of late-stage tumor progression in which epithelial cells in breast tissue dedifferentiate to more malignant cells. Furthermore, increased expression of aromatase, the rate-limiting enzyme that catalyzes the conversion of androgens to estrogens, has been observed in the adipose tissue of mammary glands in obese women with breast cancer [18]. As both adipose tissue and breast cancer epithelium express aromatase, levels of circulating estradiol are especially elevated in overweight or obese women with breast cancer [18]. This has particular clinical significance for women with ER-positive disease, as increased aromatase expression has been demonstrated to contribute to poorer prognosis in obese breast cancer patients [20].

In addition to adiposity, dietary fat is postulated to contribute to carcinogenesis through the synthesis of certain eicosanoids or lipid compounds metabolized from fatty acids that support tumor growth [12]. In diets high in animal fat, the abundance of omega- 6 fatty acids can be used in the production of eicosanoids that promote inflammation and tumor angiogenesis. Prostaglandin $\mathrm{E}_{2}\left(\mathrm{PGE}_{2}\right)$ is the most abundant proinflammatory eicosanoid identified in breast cancer and has been shown to stimulate tumor cell proliferation through aromatase upregulation [21]. As aromatase is encoded by the 
CYP19 gene, $\mathrm{PGE}_{2}$ stimulates CYP19 transcription, increasing aromatase expression and bioavailable estrogen. Ultimately, this manifests as increased expression of estrogen receptor target genes [11]. Collectively, these findings provide evidence for the association between diet-induced adiposity and the promotion of tumorigenesis, reinforcing targeted reductions in dietary fat intake.

\section{Low-Fat, High Fruit, and Vegetable Diet and Risk of Breast Cancer Recurrence}

Similar to the WINS [7], the WHEL study [8] was driven by early epidemiological evidence suggesting a lower risk of breast cancer with a low-fat diet, which the investigators combined with preclinical evidence supporting the anticarcinogenic properties of plant-derived foods [22]. The WHEL study $(n=3088)$ recruited women aged 18-70 diagnosed with operable invasive breast carcinoma (AJCC version VI Stage I, II or III) who had completed surgery and radiotherapy but not chemotherapy. The intervention group $(n=1537)$ received telephone counseling, cooking classes, and a newsletter promoting a daily intake of 5 vegetable servings, 16 oz vegetable juice, 3 fruit servings, $30 \mathrm{~g}$ fiber, and 15-20\% energy from fat. The comparison group $(n=1551)$ received written dietary guidelines of 5 servings of fruit and vegetables. Over the mean 7.3-year follow-up, $16.7 \%$ of women in the intervention group experienced a recurrence event compared to $16.9 \%$ of women in the comparison group (adjusted HR $=0.96$; $95 \% \mathrm{CI}, 0.80$ to $1.14 ; p=.63$ ), while $10.1 \%$ of women in the intervention group died compared to $10.3 \%$ in the comparison group (adjusted $\mathrm{HR}=0.91 ; 95 \% \mathrm{CI}, 0.72$ to 1.15 ; $p=.43$ ). No significant differences in weight as a result of the intervention were observed.

From these findings, the authors concluded that a low-fat, high vegetable, and fruit intake diet did not reduce recurrence events or provide survival benefit to women with early-stage breast cancer. However, in a secondary analysis of 2967 WHEL participants [23], breast cancer survivors without hot flashes (HF-negative) in the diet group had $31 \%$ fewer recurrence events compared to HF-negative women in the comparison group (adjusted $\mathrm{HR}=0.69 ; 95 \% \mathrm{CI}, 0.51$ to $0.93 ; p=.02$ ). The effect was even stronger in HF-negative post-menopausal women, as a $47 \%$ reduction in risk was identified compared to HF-negative women in the comparison group. As HFs have been associated with lower estrogen levels in women undergoing breast cancer therapy, including tamoxifen or aromatase inhibitors, HF-negative women have been found to have higher estrogen levels and an increased risk of recurrence [24]. Thus, the reduced risk of recurrence observed in HF-negative women in the diet intervention group compared to HF-negative women in the comparison group was attributed to lower estrogen levels from the low-fat, high fruit, and vegetable diet [23]. In a third follow-up analysis of the WHEL data, Thomson et al. [25] examined the association between cruciferous vegetable intake and risk of recurrence among tamoxifen users $(n=1765)$ and found that women who were in the highest reported vegetable intake tertile at baseline had overall lower hazard for breast cancer recurrence $(\mathrm{HR}=0.48 ; 95 \%$ CI 0.32-0.70; $p=0.005)$.

\section{Low-Fat, High Fruit, and Vegetable Diet and Risk of Breast Cancer Recurrence: Plausible Mechanisms}

The postulated mechanisms underlying a high intake of fruits and vegetables in reducing the risk of recurrence are related to the role of plant-derived foods as a source of chemopreventive compounds and having the ability to lower circulating estrogen concentrations. The anticarcinogenic properties of such a dietary pattern have been attributed to high levels of phytonutrients, including carotenoids, polyphenols, and isothiocyanates [22]. Preclinical data investigating the cancerpreventative activity of phytonutrients support this hypothesis, as the carotenoid lycopene was demonstrated to inhibit IGF-1-induced [26] and estrogen-induced proliferation in breast cancer cells [27]. Yet, despite evidence for the inhibitory action of phytonutrients on breast cancer cell proliferation, and observational data supporting an inverse relationship between phytonutrient consumption and risk of recurrence [25], phytonutrient interventions demonstrating actual inhibition of estrogen- and IGF-1 dependent signaling in breast cancer patients have yet to be conducted.

The second mechanism by which diet composition may influence disease recurrence and survival is through an effect on reproductive hormones. A high-vegetable, high-fiber, low-fat diet intervention has been associated with a significant reduction in serum estradiol concentrations in women diagnosed with early-stage breast cancer, even without loss of body weight [28]. However, fiber intake was also found to be independently related to reductions in serum estradiol concentrations, suggesting that increased fiber, rather than decreased fat intake, may have been responsible for reductions in circulating estrogen in these breast cancer patients. The biological mechanism supporting the role of fiber in positive breast cancer outcomes is related to its ability to bind to estrogen, as fiber supplementation in female rats with mammary tumors resulted in increased fecal excretion and inhibition of intestinal reabsorption of estrogen [29]. Taken together, these results suggest a potentially beneficial influence of fiber on breast cancer recurrence due to reductions in circulating estrogen. However, as no direct evidence exists to support this conclusion, interpretation of the epidemiological data is limited.

\section{Soy and Vitamin D Intake and Risk of Breast Cancer Recurrence}

At present, no RCTs have been conducted expressly incorporating soy or vitamin $\mathrm{D}$ as part of a diet intervention; therefore, 
in the proceeding section, observational and preclinical data are discussed in relation to breast cancer survival.

Conflicting data exists between epidemiological and preclinical investigations on the role of soy intake in breast cancer recurrence. While the epidemiological data has found no association between soy intake and adverse breast cancer events [30-34], the in vitro and in vivo data have raised concerns on the safety of phytoestrogens in breast cancer survival. Soy foods, such as soy milk and tofu, are rich in isoflavones that are structurally similar to the primary endogenous estrogen $17 \beta$-estradiol [32]. A major isoflavone in soy products is genistein, which can compete for binding to the ER in breast tissue, although the binding affinity of isoflavones for the ER is a magnitude lower than that of $17 \beta$-estradiol [35]. As such, isoflavones have traditionally been regarded as ER modulators that can exhibit estrogen-like properties, potentially promoting mammary tumorigenesis [30]. However, the concerns surrounding the relationship between genistein and breast tumor proliferation have been questioned in a recent review, with evidence cited to support the anticarcinogenic effects of genistein while disputing its stimulatory effects [35]. For example, in a DMBA (7,12-dimethylbenz[a]anthracene) model of induced mammary carcinoma, female rats exposed to genistein during the early neonatal period had reduced incidence of mammary tumors compared to control rats [36], while in an orthotopic model of breast cancer, mice who received genistein supplementation exhibited a 10-fold lower metastatic burden compared to control mice [37]. Yet, the authors acknowledge preclinical data reinforcing the stimulatory effect of genistein on established ER-positive mammary tumors, albeit at a rate lower to estradiol. In contrast, the epidemiological data unequivocally refutes the dangers of isoflavones in breast recurrence, with data from 4 large clinical trials reporting no adverse relationship between soy intake and breast cancer prognosis [30-32, 34], and 1 observational trial in fact reporting a statistically significant reduced risk of recurrence with a post-diagnosis soy food consumption $\geq 10 \mathrm{mg}$ isoflavones/day $(\mathrm{HR}=0.75 ; 95 \% \mathrm{CI}$ 0.61-0.92) [33]. Thus, despite the extensive research investigating the adverse effect of isoflavones on mammary tumorigenesis, it appears from observational data that soy-derived genistein does not negatively impact breast cancer recurrence, particularly in ER-negative disease. However, before clinical guidelines can be proposed, RCTs incorporating soy products in a dietary intervention are warranted to demonstrate clear evidence that soy intake is safe and possibly chemopreventive.

Similarly, investigations on the relationship between vitamin D intake and breast cancer recurrence have been limited to preclinical and observational data, with additional insight provided by correlative studies nested in RCTs comprised of women with early-stage breast cancer. Though evidence from in vitro, animal, and observational studies support the role of vitamin $\mathrm{D}$ in improving breast cancer outcomes, data from prospective [38] and observational studies nested randomized trials $[39,40 \cdot 41]$ in women with breast cancer have not suggested prognostic associations $[39,40 \bullet, 41]$. The physiological mechanism underpinning the inhibitory action of vitamin D on breast cancer centers on calcitriol, the hormonally active form of vitamin D. Calcitriol inhibits proliferation of human breast cancer cell lines and arrests tumor growth in xenograft models through several processes that ultimately suppress estrogen pathways in breast cancer cells (reviewed in Krishnan et al. [42]). Briefly, calcitriol reduces estrogen synthesis through direct repression of CYP19 and through decreases in $\mathrm{PGE}_{2}$. In addition to reducing estrogen synthesis, calcitriol downregulates ER $\alpha$ expression, suppressing the action of estrogen. The mechanistic evidence is in agreement with observational data supporting an adverse association between breast cancer recurrence and levels of serum 25hydroxyvitamin $\mathrm{D}[25(\mathrm{OH}) \mathrm{D}]$, the precursor to calcitriol $[43,44]$. In contrast, findings from observational studies contradict these results, as no associations between serum $25(\mathrm{OH}) \mathrm{D}$ levels and breast cancer outcomes have been observed in a secondary analysis of the WHEL data [38], or in adjuvant (MA.21 [40•], MA.14 [41]) or neo-adjuvant (I-SPY [39]) settings. Although the nesting of observational studies in randomized trials such as that in Clark et al. [39], Lohmann et al. [40•], and Pritchard et al. [41] has its limitations, the RCTs provide greater standardization of tumor, treatment, and outcome characterizations than the non-therapeutic observational studies. Clearly, therapeutic interventions involving vitamin D supplementation would be desirable in evaluating the effects of vitamin D on breast cancer outcomes, as neither observational nor post hoc correlative studies provide substantive evidence on which to base recommendations.

\section{Summary and Current Dietary Recommendations}

Though results from the large-scale WHEL and WINS randomized trials do not support an effect of low-fat/high fruit and vegetable diets on overall survival, evidence from WINS and a secondary analysis of the WHEL data have potentially demonstrated a benefit of a reduced-fat diet on risk of recurrence. As the positive findings from WINS and the secondary analyses of WHEL were associated with weight loss or hormonal status, the mechanism by which a reduced-fat diet may exert its beneficial effects may be mediated through metabolic hormones and factors associated with decreased adiposity. Furthermore, although data from randomized trials is lacking, soy intake does not appear to be adversely related to breast cancer outcomes nor are low vitamin D levels unequivocally associated with recurrence or increased mortality. Despite the limited evidence supporting diet interventions in preventing breast cancer recurrence, recent recommendations for cancer survivors from the American Cancer Society (ACS) [6] underscore the growing importance of a healthy diet across the 
continuum of cancer survivorship. As weight gain is a common occurrence following breast cancer diagnosis, and survivors are at increased risk for comorbid conditions such as cardiovascular disease and diabetes, achieving a healthy weight through reductions in caloric intake and increases in energy expenditure through a combination of diet, exercise, and behavioral strategies is encouraged (Fig. 1). In general, the ACS diet recommendations follow guidelines similar to that of the American Heart Association [45], with a recommended dietary composition of 45-65\% energy from carbohydrate, $10-35 \%$ of energy from protein, and $20-35 \%$ energy from fat. Reductions in the energy density of the diet by limiting portions of energy-dense foods, such as high-fat and sugary items, are emphasized, in addition to increasing intake of low-energy-dense foods, such as vegetables and fruits. Furthermore, consuming carbohydrate sources that are rich in essential nutrients, phytochemicals, and fiber are encouraged, while refined grains, such as white bread and white process, should be avoided. As protein intake is essential during all stages of survivorship, the ACS recommends consumption of protein that is low in saturated fat and limited intake of processed meats. However, not all fat is adverse, as intake of omega-3 fatty acids from sources like fish or nuts is advised. In terms of dietary supplements, nutrients should be primarily obtained through food sources, with supplementation only considered if a nutrient deficiency is clinically present. These diet recommendations are summarized in Table 1.

\section{Physical Activity/Exercise and Risk of Breast Cancer Recurrence}

For the purposes of this review, physical activity (PA) is defined as any movement requiring energy expenditure, while exercise is PA that is planned, structured, and directed to

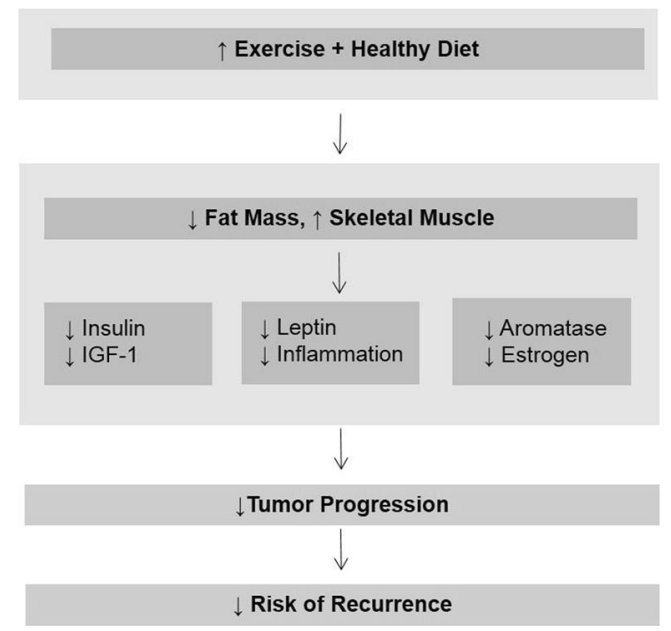

Fig. 1 Plausible mechanisms mediating the impact of diet and exercise on breast cancer recurrence. $I G F-1$ insulin-like growth factor-1 enhancing physical fitness and health [4]. "PA" will be used when referencing epidemiologic studies and "exercise" when referring to RCTs.

An increased risk of breast cancer recurrence in breast cancer survivors $(\sim 34 \%)$ compared to physically active breast cancer survivors has been, in part, attributed to sedentary lifestyle behaviors [46]. Since exercise has been shown to elicit numerous health benefits including improved quality of life, increased physical functioning, reduced cancer-related fatigue as well as reductions in obesity-related comorbidities in cancer survivors [47-49], the plausible effects of exercise on breast cancer recurrence are important to consider. Although there have been efforts to ameliorate the side effects breast cancerrelated treatments with increased aerobic and resistance exercise [50-52], the evidence is lacking to provide information on the effects of exercise on breast cancer recurrence. This section presents evidence from both epidemiological and clinical trials examining the effect of PA or exercise on breast cancer recurrence outcomes. In addition, possible mechanisms elucidating the relationship between PA/exercise and disease recurrence will be discussed.

\section{PA and Risk of Breast Cancer Recurrence: Epidemiologic Evidence}

Previously published review articles have summarized the effects of PA on breast cancer events and mortality [3, 5, 53], including the recent systemic review summarized here [54•]. This systemic review and meta-analysis included 22 prospective studies and, in brief, found an inverse relationship between PA and all-cause, breast cancer-related death and breast cancer events (i.e., recurrence). For example, compared to those who reported low/no lifetime recreational prediagnosis PA, women who reported high lifetime recreational pre-diagnosis PA levels had a significantly lower risk of allcause $(\mathrm{HR}=0.82,95 \% \mathrm{CI} 0.70-0.96, p<0.05)$ and breast cancer-related death $(\mathrm{HR}=0.73,95 \%$ CI $0.54-0.98, p<$ $0.05)$. Significant risk reductions for all-cause and breast cancer-related death was observed for more recent $(\leq 12$ years) pre-diagnosis recreational $\mathrm{PA}(\mathrm{HR}=0.73,95 \% \mathrm{CI} 0.65-0.82$, $p<0.001$; and $\mathrm{HR}=0.84,95 \%$ CI $0.73-0.97, p<0.05$, respectively), post-diagnosis PA ( $\mathrm{HR}=0.52,95 \%$ CI 0.43 $0.64, p<0.01$; and $\mathrm{HR}=0.59,95 \%$ CI $0.45-0.78, p<0.05$, respectively), and meeting recommended PA guidelines (i.e., $\geq 8 \mathrm{MET}$-h/week; MET or metabolic equivalent, is a concept used to express the energy cost of physical activities as a multiple of resting metabolic rate where 1 MET if the amount of oxygen consumed while sitting at rest) post-diagnosis $(\mathrm{HR}=0.54,95 \%$ CI $0.38-0.76, p<0.01$; and $\mathrm{HR}=0.67$, $95 \%$ CI $0.50-0.90, p<0.01$, respectively). Pre-diagnosis (lifetime and more recent combined) and post-diagnosis PA were associated with reduced risk of breast cancer events (breast cancer progression, new primaries and recurrence 
Table 1 Diet and exercise recommendations for breast cancer survivors implicated to target reduced risk of recurrence [6]

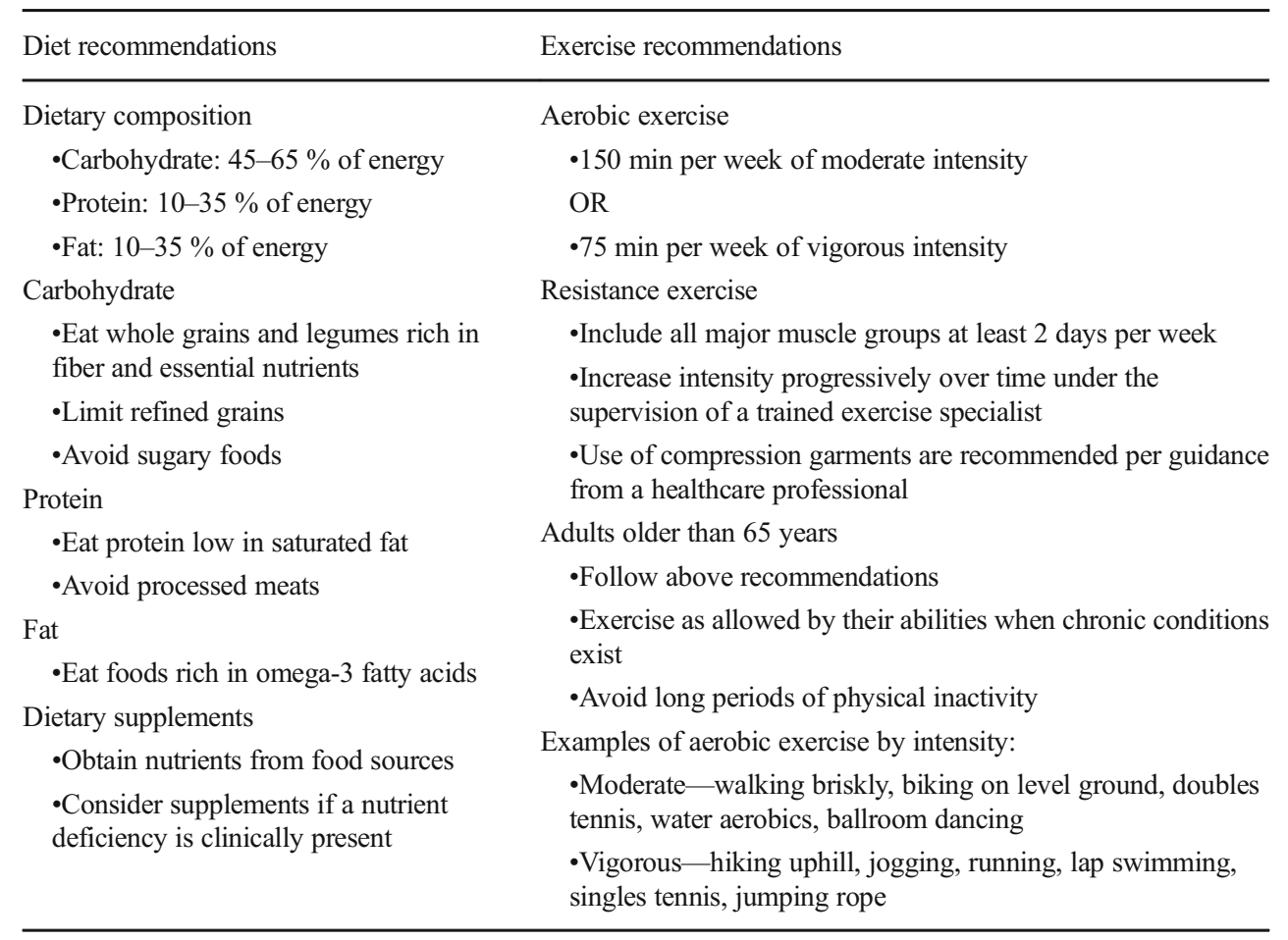

combined $)(\mathrm{HR}=0.7295 \% \mathrm{CI} 0.56-0.91, p<0.01$; and $\mathrm{HR}=0.79$, $95 \%$ CI $0.63-0.98, p<0.05$, respectively). Thus, the authors suggest this evidence supports the notion that exercise is an important consideration for reducing these events in breast cancer survivors.

One of the hallmark epidemiological studies to establish the association of PA and survival after breast cancer diagnosis was the Nurses' Health Study [55]. This prospective observational study examined breast cancer mortality risk according to PA category $(<3,3-8.9,9-14.9,15-23.9$, or 24 metabolic equivalent task [MET] hours per week) and included 2987 women diagnosed with stage I-III breast cancer. When compared with women who engaged in $<3$ MET-h/ week of PA, the adjusted RR of death from breast cancer was 0.80 (95\% CI, 0.60-1.06) for 3-8.9 MET-h/week; 0.50 (95\% CI, 0.31-0.82) for 9-14.9 MET-h/week; 0.56 (95\% CI, 0.38-0.84) for 15-23.9 MET-h/week; and 0.60 (95\% CI, $0.40-0.89)$ for 24 or more MET-h/week $\left(\mathrm{P}_{\text {trend }}=.004\right)$. PA was particularly beneficial among women with hormoneresponsive tumors where the RR of breast cancer death for women with hormone-responsive tumors who engaged in $\geq 9$ MET-h/week of PA compared with women with hormoneresponsive tumors who engaged in $<9$ MET-h/week was 0.50 (95\% CI, 0.34-0.74). The absolute unadjusted mortality risk reduction was $6 \%$ at 10 years for women who engaged in $\geq 9$ MET-h/week when compared with women who engaged in $<3$ MET-h/week of PA. The authors concluded that PA after breast cancer diagnosis may reduce the risk of death from the disease, with the greatest benefit observed in women who performed the equivalent of walking 3-5 h/week at an average pace (2$2.9 \mathrm{mph}$ ). Thus, breast cancer survivors who participate in regular exercise may improve their survival.

In summary, epidemiological evidence supports the participation in PA before and after breast cancer diagnosis as a contributing factor in decreasing risk of breast cancer recurrence and mortality. Future clinical trials are warranted to establish the underlying mechanisms as purported below.

\section{Exercise and Risk of Breast Cancer Recurrence: Evidence from Clinical Trials}

The effect of exercise on breast cancer recurrence is an area currently under much speculation yet evidence from RCTs is lacking. However, pertinent to this area is the Supervised Trial of Aerobic versus Resistance Training (START) multicenter Canadian RCT which recently reported an exploratory analysis of follow-up cancer outcomes in breast cancer survivors [56••]. The START trial randomized 242 breast cancer survivors to usual care, supervised aerobic, or resistance training while undergoing adjuvant chemotherapy. For this analysis, the 2 exercise groups were combined $(n=160)$ to examine the effect on disease-free survival (DFS). Eight-year DFS was lower for the exercise groups $(82.7 \%)$ compared with the usual care group (75.6\%; HR, 0.68: $95 \%$ CI, 0.37-1.24; log-rank, $P=0.21)$. Subgroup analyses resulted in potentially stronger effects of exercise on DFS for women who were overweight/obese (HR, 0.59: $95 \%$ CI, 0.27-1.27), had stage II/III cancer (HR, 0.61: $95 \%$ CI, 0.31-1.20), estrogen 
receptor-positive tumors (HR, 0.58: $95 \%$ CI, 0.26-1.29), human epidermal growth factor receptor 2-positive tumors (HR, 0.21: $95 \%$ CI, 0.04-1.02), received taxane-based chemotherapies (HR, 0.46: $95 \% \mathrm{CI}, 0.19-1.15$ ), and completed $\geq 85 \%$ planned chemotherapy (HR, 0.50: $95 \%$ CI, 0.25-1.01). This is the first randomized data, to our knowledge, to suggest the addition of exercise to standard chemotherapy as a means to improve breast cancer outcomes and a Phase III trial is warranted to further investigate this area.

In addition to the START trial, our laboratory is currently conducting an RCT to investigate the effects of a 16-week supervised combined aerobic and resistance exercise intervention on metabolic syndrome and DFS in overweight/obese breast cancer survivors who completed chemotherapy and/or radiation within the prior 6 months [57]. Although the main outcomes of this study include metabolic syndrome and related cardio-metabolic outcomes, a follow-up analysis is included to assess DFS annually for 10 years after completion of the intervention. This ongoing RCT currently has 79 breast cancer survivors enrolled (as of March 2016), and we seek to enroll a total of 200 women for our DFS analysis. We hope this investigation contributes to the exploratory hypothesis that exercise may affect breast cancer prognosis.

\section{PA and Exercise and Risk of Breast Cancer Recurrence: Plausible Mechanisms}

The physiological mechanisms through which physical activity may influence risk of breast cancer recurrence relate to the effect of exercise on adipose tissue and skeletal muscle [58] and is summarized in Fig. 1. By targeting adiposity, exercise may reduce adverse outcomes associated with fat accumulation including an altered hormonal environment and overexpressed adipokine production, both of which may act to promote tumor development and growth [10]. In addition, increases in skeletal muscle as a result of exercise may reduce insulin resistance, a common comorbidity in obese individuals [59].

Metabolic hormones are adversely affected by adiposity, with detrimental alterations observed in insulin and IGF-1. Unfavorable changes in the hormonal milieu provide an optimal environment for breast cancer cell growth, as insulin and IGF-1 have been shown to promote tumor development and metastases via signaling through their receptors [13•]. In addition, insulin and IGF-1 increase levels of bioavailable estrogen via aromatase activity in adipose tissue and through reductions in circulating concentrations of sex hormone binding globulin (SHBG), a protein that binds to estrogen to decrease its bioavailability [60]. To date, 4 RCTs utilizing exercise interventions with metabolic hormones as primary endpoints have been conducted in breast cancer survivors [61-64], yet only 3 have demonstrated that chronic exercise training can improve insulin [63] or IGF-1 levels [61, 62], even in the absence of fat loss [63]. One possible explanation for the differences in efficacy between Schmitz et al. [64] and the 3 interventions can be attributed to exercise prescription, as the 3 interventions met the American College of Sports Medicine (ACSM)/ACS exercise recommendation for cancer survivors of at least $150 \mathrm{~min} /$ week of moderate intensity exercise or $75 \mathrm{~min} /$ week of vigorous intensity exercise [6]. In addition, the 3 interventions employed aerobic training (AT), with Ligibel et al. [63] utilizing a combined program of AT and resistance training (RT), while Schmitz et al. [64] employed only RT. Another explanation for differing results between the investigations is the enrollment of sedentary, obese breast cancer survivors in the studies of Irwin [62] and Ligibel [63] compared to the leaner and more active women in Schmitz's study. Baseline concentrations of insulin were lower in Schmitz's participants; hence, exercise interventions may have greater effect in less active breast cancer survivors with higher adiposity. Yet, Schmitz et al. also reported significant increases in skeletal muscle following RT, which conceivably could contribute to improvements in whole-body glucose tolerance and insulin resistance [59]. However, Schmitz et al. observed no changes in insulin resistance following RT, suggesting that future exercise interventions in breast cancer survivors should incorporate higher intensities, greater volume or employ AT in addition to RT to elicit optimal changes in cardio-metabolic variables.

While increased concentrations of insulin and IGF-1 may affect estrogen levels, as previously discussed in the diet section, bioavailable estrogen is also independently influenced by adiposity. Elevated levels of circulating estrogens have been attributed to excess adipose tissue and increased expression of aromatase, the enzyme that catalyzes estrogen synthesis, in the breast tissue of obese breast cancer survivors [18]. As estrogenic stimulation has been shown to promote tumorigenesis and breast cancer pathogenesis, higher adiposity has been suggested to contribute to poorer prognosis in obese breast cancer patients [20]. Exercise may favorably alter bioavailable estrogen through either mechanism, although separating the effects of fat loss from reductions in insulin/IGF-1 due to exercise has not yet been elucidated in breast cancer survivors. Rock et al. [60] demonstrated significant reductions in bioavailable estrogen in post-menopausal breast cancer survivors following a combined physical activity and diet intervention. As this finding was observed alongside significant weight loss of $5 \%$, increased SHBG, and decreased insulin and leptin concentrations, the authors hypothesized that the reductions in estrogen were likely mediated by fat loss.

Leptin alone has been associated with a more aggressive tumor type in post-menopausal breast cancer survivors with ER-positive disease [65]. As leptin is secreted from white adipose tissue, higher circulating levels have been observed in overweight/obese breast cancer survivors compared to lean patients [66・•]. Leptin promotes the recruitment of macrophages, which in turn, release pro-inflammatory cytokines, contributing to a chronic state of systemic inflammation that 
has been purported to correlate with poorer prognosis in breast cancer survivors [67]. As such, an intervention that reduces adiposity would be expected to decrease leptin concentrations alone or in combination with reductions in macrophageproduced inflammatory cytokines. Evidence supporting the use of exercise training in lowering leptin concentrations in breast cancer survivors is emerging, with one RCT demonstrating significant improvements in leptin in overweight breast cancer survivors following a 3-month intervention of $150 \mathrm{~min} /$ week of exercise, although non-significant changes were observed in the inflammatory markers IL-6, tumor necrosis factor (TNF)- $\alpha$, and IL-10 [67]. This finding suggests that leptin levels in breast cancer survivors are responsive to exercise, and if leptin is indeed related to recurrence, then exercise may be a useful therapeutic in improving breast cancer outcomes. Future investigations are needed to determine the role of exercise and its potential mechanisms in favorably altering adipokine concentrations in breast cancer survivors.

\section{Summary and Current Exercise Recommendations}

Epidemiological evidence supports the participation in PA before and after breast cancer diagnosis as a contributing factor in decreasing risk of breast cancer recurrence and mortality. Supportive data from RCTs to promote exercise as a means to reduce disease recurrence is lacking; however, promising data from the sole RCT reviewed here examining the effects of exercise on disease outcomes [56] suggests that there may be beneficial effects of exercise on breast cancer recurrence. Additional RCTs are necessary to guide specific exercise recommendations to target disease recurrence.

Current recommendations set forth by ACSM [68], ACS [69], and the US Department of Health and Human Services [70] (Table 1) encourage cancer survivors to follow exercise prescription for healthy age-matched adults (aged 1864 years) which includes 150 min per week of moderate or $75 \mathrm{~min}$ per week of vigorous aerobic exercise. Musclestrengthening activities or resistance exercise involving all major muscle groups should be performed 2 days per week; however, these exercises should be led by a trained exercise specialist to ensure the intensity is progressed appropriately. Use of compression garments during exercise is recommended per guidance from a healthcare professional.

\section{Combined Diet and PA and Risk of Breast Cancer Recurrence}

Due to the independent physiologic effects of diet and physical activity on adiposity, inflammation, and hormonal regulation, a more potent stimulus to target breast cancer recurrence may involve a combined diet and physical activity intervention. In this section, we will discuss the few clinical [71••, 72, 73] and epidemiologic [74] investigations assessing a combined diet and physical activity intervention on breast cancer recurrence or survival.

\section{Combined Diet and PA and Risk of Breast Cancer Recurrence: Epidemiologic Evidence}

Although the focus of this manuscript is on lifestyle intervention effects on breast cancer recurrence, it is important to recognize a hallmark epidemiologic study including combined diet and PA as it influences mortality due to the long-term observations afforded by this study. This includes the WHEL study [8] previously mentioned in the diet portion of this review that will be expanded upon here as it pertains to diet and PA [74].

The WHEL is a prospective cohort study including 1490 women treated for early-stage breast cancer between 1991 and 2000 who enrolled approximately 2 years post-diagnosis. Interactions between lifestyle factors and mortality were examined. Dietary patterns were assessed at baseline using 2 24-h dietary recalls on random days during a 3-week period using a telephone-based dietary assessment with associated analysis software. PA was assessed at baseline using a 9-item questionnaire on usual PA with queries on frequency, duration, and intensity used to determine metabolic equivalent (METs) values.

Importantly, reduced mortality was weakly associated with higher fruit and vegetable consumption $(p=0.02)$, increased PA $(p=0.02)$, and normal BMI $(p=0.06)$. Using a Cox multivariate model, the combination of consuming 5 or more daily servings of fruits and vegetables, and accumulating 540+ MET tasks-min/week (or walking $30 \mathrm{~min} 6$ days/week) was associated with improved survival $(\mathrm{HR}=0.56 ; 95 \% \mathrm{CI}, 0.31$ to $0.98, p=$ 0.04 ). Notably, the approximate $50 \%$ reduced risk was observed in both obese and non-obese women. Univariate analysis demonstrated that high fruit and vegetable intake and high PA levels were advantageous on survival for hormone receptor-positive cancers (ER-positive, PR-negative; $p=0.04$; ER-positive, PRpositive; $p=0.01$ ). The latter result suggests the mechanism of action may involve reproductive gonadal hormones as previously suggested for diet and PA lifestyle factors on prognosis.

Despite the observational nature of the WHEL study, it emphasizes the relation between lifestyle factors and disease prognosis in a large cohort of women with early-stage breast cancer. These results have since been used to implement intervention trials as will be discussed in the subsequent section.

\section{Combined Diet and PA and Risk of Breast Cancer Recurrence: Evidence from Clinical Trials}

As obesity is a major risk factor breast cancer recurrence and morbidity in pre- and post-menopausal women $[5,75,76]$, targeting reductions in body weight through combined diet and exercise interventions have been conducted. Three RCTs will be 
discussed, including the Exercise and Nutrition to Enhance Recovery and Good Health for You (ENERGY) trial [71••], a smaller yet pertinent RCT from Scott and colleagues [72], and an ongoing RCT - the Diet and Androgens (DIANA)-5 study [73].

The ENERGY trial is the largest weight loss intervention trial among breast cancer survivors to date [71••]. This multicenter trial included 692 overweight/obese women who were approximately 2 years from primary treatment for early-stage breast cancer. Women were randomized to either a groupbased behavioral intervention with telephone counseling and newsletters to support weight loss or a less intensive control intervention including weight management resources and materials. The goal of the intervention was a $7 \%$ weight loss in 2 years and included weekly 1 -h group sessions for the first 4 months tapering to every other week for 2 months followed by monthly from month 6 onward. Dietary guidance promoted a reduction in energy intake of 500-1000 kcal/day and a PA goal of an average of $60 \mathrm{~min} /$ day at a moderate intensity. Body weight was measured at $6,12,18$, and 24 months.

At 12 months, the intervention group experienced a mean weight loss of $6.0 \%$ of initial weight compared to $1.5 \%$ in the control group $(p<.001)$. At 24 months, the group differences remained significant $(p<.001)$ with mean weight loss of 3.7 and $1.3 \%$ for the intervention and control groups, respectively. This finding implies that a behavioral weight loss intervention can result in clinically meaningful weight loss in this population. Although the authors are unable to report the effect of the intervention on recurrence and survival at this point in time, they report that a 4-6\% weight loss substantially reduces circulating levels of estrogens and cytokines, which are implicated mediators of breast cancer recurrence.

Scott et al. (2013) examined the effects of a diet and PA intervention on body weight in overweight breast cancer survivors in an effort to determine the relationship between body weight and biomarkers associated with disease recurrence and survival [72]. Ninety women treated for early-stage breast cancer in the previous 3-18 months were randomly assigned to a 6month exercise and hypocaloric healthy eating program or control group. The physical activity portion included 3 weekly 30 min aerobic exercise (65-85\% age-predicted heart rate maximum) sessions followed by 10-15 min of muscle-strengthening exercises using resistance bands, hand weights and stability balls under supervision. The dietary intervention included one-on-one individualized dietary advice and written information pertaining to portion sizes and a healthy eating plan. The goal of the dietary intervention was a reduction in total daily caloric intake by $600 \mathrm{kcal}$ from their calculated energy requirements. Collectively, the authors purported that this intervention would result in an estimated weight loss of up to $0.5 \mathrm{~kg} /$ week.

The intervention resulted in moderate weight loss (median difference from baseline of $-1.09 \mathrm{~kg} ; p=0.07$ ) in the intervention group and a significant reduction in waist circumference $(p$ $<0.001)$. Biomarkers associated with long-term prognostic outcomes of breast cancer including leptin $(p=0.005)$, total cholesterol ( $p=0.046)$, and HDL ( $p=0.015)$ were significantly altered. Positive changes in leptin and total cholesterol were significantly associated with reductions in body weight and waist circumference ( $p<0.01 ; R$ values not reported by authors). The authors concluded that a 6-month exercise and hypocaloric eating program positively impacted health outcomes (i.e., weight, waist circumference, leptin) that influence long-term prognosis in overweight breast cancer survivors.

These 2 RCTs provide strong implications for the impact of combined diet and exercise on breast cancer recurrence with the ENERGY trial relying on home-based interventions and Scott's trial including supervised, clinic setting interventions. Both trials did not directly assess survival rates yet rather measured outcomes strongly linked to prognosis such as central adiposity, sex hormones, lipid profiles, and proinflammatory biomarkers, which have been discussed previously in this manuscript. Each trial provides evidence of strong effects of a combined intervention on weight loss with their respective study designs, thus implicating the need for future RCTs with long-term follow-up assessments to link these changes with disease recurrence.

A notable ongoing RCT is the Diet and Androgens (DIANA)-5 study, which is a multicenter RCT examining the effectiveness of a Mediterranean diet and PA intervention on breast cancer events between 2008 and 2010, followed through 2015 [73]. The intervention consists of a counseling program with cooking classes, exercise sessions, and reinforcing meetings and print materials. The PA goals are to participate in a moderate intensity PA program of $210 \mathrm{~min} /$ week (25 METs) over at least 3 days/week and to decrease sedentary behavior by $30 \mathrm{~min} /$ day on at least 5 days/week. During the first 12 months, 1 group PA session/month is offered to increase PA adoption. The diet program focuses on reducing glycemic and insulinemic responses through (1) moderate caloric restriction through increased consumption of highly satiating foods (refined cereals, legumes, and vegetables), (2) reduced consumption of high glycemic index food, (3) reduced consumption of saturated fat, and (4) reduced protein intake, particularly animal protein (except fish). Outcomes include recurrence, death from breast cancer, and death from any cause. The investigators seek to establish a comprehensive lifestyle modification to increase breast cancer event-free survival. The results of such a trial may be the first to establish breast cancer outcomes affected by a diet and PA intervention.

\section{Summary}

Despite the lack of concrete epidemiologic and clinical evidence supporting the effects of combined diet and PA lifestyle modifications on breast cancer recurrence, the aforementioned studies emphasize the benefits of such behaviors on health 
outcomes biologically linked to recurrence. Further, these studies shed light on the need to study diet and exercise interventions moving forward to specifically define parameters to guide the adoption of lifestyle behaviors targeting disease-free survival. In the interim, dietary and exercise guidelines outlined in Table 1 provide a comprehensive foundation that breast cancer survivors can rely upon to adopt a healthier lifestyle during survivorship.

\section{Conclusions}

In evaluating evidence from RCTs involving diet, exercise, or combined diet and exercise interventions, the most consistent finding was that reductions in adiposity accompanied the most beneficial changes in breast cancer outcomes, including survival, risk of recurrence, or biomarkers associated with prognosis. These data suggest that body composition is a crucial, modifiable risk factor for breast cancer prognosis, and interventions targeting fat loss and skeletal muscle gain may yield improvements that translate to clinically meaningful outcomes. While data from currently conducted RCTs is still emerging, a combined intervention of diet and exercise appears to hold the most promise in modifying the incidence of additional breast cancer events or mortality and is also reinforced by epidemiological evidence. Yet, the lack of conclusive evidence supporting whether exercise- or diet-induced changes can occur independent of changes in fat mass necessitates future work examining the complex interaction between adiposity, energy balance, and breast cancer recurrence. Regardless, the data evaluated in this review support the use of both dietary modification and increased exercise as a means of improving overall health and reducing risk of recurrence and mortality in breast cancer survivors.

\section{Compliance with Ethical Standards}

Conflict of Interest Christina M. Dieli-Conwright, Kyuwan Lee, and Jacqueline L. Kiwata declare that they have no conflict of interest.

Human and Animal Rights and Informed Consent This article does not contain any studies with human or animal subjects performed by any of the authors.

\section{References}

Papers of particular interest, published recently, have been highlighted as:

- Of importance

•- Of major importance

1. Siegel RL, Miller KD, Jemal A. Cancer statistics. Ca-Cancer J Clin. 2015;65(1):5-29.
2. Demark-Wahnefried W, Campbell KL, Hayes SC. Weight management and its role in breast cancer rehabilitation. Cancer. 2012;118(8 Suppl):2277-87.

3. Davies NJ, Batehup L, Thomas R. The role of diet and physical activity in breast, colorectal, and prostate cancer survivorship: a review of the literature. Br J Cancer. 2011;105 Suppl 1:S52-73.

4. Marmot M, Atinmo T, Byers T, Chen J, Hirohata T, Jackson A, James W, Kolonel L, Kumanyika S, Leitzmann C: Food, nutrition, physical activity, and the prevention of cancer: a global perspective. 2007

5. Patterson RE, Cadmus LA, Emond JA, Pierce JP. Physical activity, diet, adiposity and female breast cancer prognosis: a review of the epidemiologic literature. Maturitas. 2010;66(1):5-15.

6. Rock CL, Doyle C, Demark-Wahnefried W, Meyerhardt J, Courneya KS, Schwartz AL, et al. Nutrition and physical activity guidelines for cancer survivors. CA Cancer J Clin. 2012;62(4): 242-74.

7. Chlebowski RT, Blackburn GL, Thomson CA, Nixon DW, Shapiro A, Hoy MK, et al. Dietary fat reduction and breast cancer outcome: interim efficacy results from the Women's Intervention Nutrition Study. J Natl Cancer Inst. 2006;98(24):1767-76.

8. Pierce JP, Natarajan L, Caan BJ, Parker BA, Greenberg ER, Flatt $\mathrm{SW}$, et al. Influence of a diet very high in vegetables, fruit, and fiber and low in fat on prognosis following treatment for breast cancer: the Women's Healthy Eating and Living (WHEL) randomized trial. Jama. 2007;298(3):289-98.

9. Chlebowski R, Blackburn G, Hoy M, Thomson C, Giuliano A, McAndrew P, Hudis C, Butler J, Shapiro A, Elashoff R: Survival analyses from the Women's Intervention Nutrition Study (WINS) evaluating dietary fat reduction and breast cancer outcome. In: ASCO Annual Meeting Proceedings: 2008; 2008: 522

10. Cohen DH, LeRoith D. Obesity, type 2 diabetes, and cancer: the insulin and IGF connection. Endocr Relat Cancer. 2012;19(5):F27-45.

11. Howe LR, Subbaramaiah K, Hudis CA, Dannenberg AJ. Molecular pathways: adipose inflammation as a mediator of obesity-associated cancer. Clin Cancer Res. 2013;19(22):6074-83.

12. Wang D, Dubois RN. Eicosanoids and cancer. Nat Rev Cancer. 2010;10(3):181-93.

13. Belardi V, Gallagher EJ, Novosyadlyy R, LeRoith D. Insulin and IGFs in obesity-related breast cancer. J Mammary Gland Biol Neoplasia. 2013;18(3-4):277-89. This review discusses the role of the insulin-family hormones, factors and receptors in obesityassociated tumor progression and metastases in breast cancer.

14. Werner H. Tumor suppressors govern insulin-like growth factor signaling pathways: implications in metabolism and cancer. Oncogene. 2012;31(22):2703-14.

15. Law JH, Habibi G, Hu K, Masoudi H, Wang MY, Stratford AL, et al. Phosphorylated insulin-like growth factor-i/insulin receptor is present in all breast cancer subtypes and is related to poor survival. Cancer Res. 2008;68(24):10238-46.

16. Gallagher EJ, LeRoith D. Obesity and diabetes: the increased risk of cancer and cancer-related mortality. Physiol Rev. 2015;95(3):727-48.

17. Huber MA, Azoitei N, Baumann B, Grunert S, Sommer A, Pehamberger $\mathrm{H}$, et al. NF-kappaB is essential for epithelialmesenchymal transition and metastasis in a model of breast cancer progression. J Clin Invest. 2004;114(4):569-81.

18. Morris PG, Hudis CA, Giri D, Morrow M, Falcone DJ, Zhou XK, et al. Inflammation and increased aromatase expression occur in the breast tissue of obese women with breast cancer. Cancer Prev Res (Phila). 2011;4(7):1021-9.

19. Sullivan NJ, Sasser AK, Axel AE, Vesuna F, Raman V, Ramirez N, et al. Interleukin-6 induces an epithelial-mesenchymal transition phenotype in human breast cancer cells. Oncogene. 2009;28(33):2940-7.

20. Subbaramaiah K, Morris PG, Zhou XK, Morrow M, Du B, Giri D, et al. Increased levels of COX-2 and prostaglandin E2 contribute to elevated aromatase expression in inflamed breast tissue of obese women. Cancer discovery. 2012;2(4):356-65. 
21. Zhou J, Suzuki T, Kovacic A, Saito R, Miki Y, Ishida T, et al. Interactions between prostaglandin $\mathrm{E}(2)$, liver receptor homologue-1, and aromatase in breast cancer. Cancer Res. 2005;65(2): 657-63.

22. Vadodkar AS, Suman S, Lakshmanaswamy R, Damodaran C. Chemoprevention of breast cancer by dietary compounds. Anti Cancer Agents Med Chem. 2012;12(10):1185-202.

23. Gold EB, Pierce JP, Natarajan L, Stefanick ML, Laughlin GA, Caan $\mathrm{BJ}$, et al. Dietary pattern influences breast cancer prognosis in women without hot flashes: the women's healthy eating and living trial. J Clin Oncol Off J Am Soc Clin Oncol. 2009;27(3):352-9.

24. Rock CL, Flatt SW, Laughlin GA, Gold EB, Thomson CA, Natarajan L, et al. Reproductive steroid hormones and recurrencefree survival in women with a history of breast cancer. Cancer Epidemiol Biomarkers Prev Publ Am Assoc Cancer Res Am Soc Prev Oncol. 2008;17(3):614-20.

25. Thomson CA, Rock CL, Thompson PA, Caan BJ, Cussler E, Flatt $\mathrm{SW}$, et al. Vegetable intake is associated with reduced breast cancer recurrence in tamoxifen users: a secondary analysis from the Women's Healthy Eating and Living Study. Breast Cancer Res Treat. 2011;125(2):519-27.

26. Nahum A, Zeller L, Danilenko M, Prall OW, Watts CK, Sutherland RL, et al. Lycopene inhibition of IGF-induced cancer cell growth depends on the level of cyclin D1. Eur J Nutr. 2006;45(5):275-82.

27. Hirsch K, Atzmon A, Danilenko M, Levy J, Sharoni Y. Lycopene and other carotenoids inhibit estrogenic activity of 17 beta-estradiol and genistein in cancer cells. Breast Cancer Res Treat. 2007;104(2):221-30.

28. Rock CL, Flatt SW, Thomson CA, Stefanick ML, Newman VA, Jones LA, et al. Effects of a high-fiber, low-fat diet intervention on serum concentrations of reproductive steroid hormones in women with a history of breast cancer. J Clin Oncol Off J Am Soc Clin Oncol. 2004;22(12):2379-87.

29. Kendall ME, Cohen LA. Effect of dietary fiber on mammary tumorigenesis, estrogen metabolism, and lipid excretion in female rats. In Vivo. 1992;6(3):239-46.

30. Caan BJ, Natarajan L, Parker B, Gold EB, Thomson C, Newman V, et al. Soy food consumption and breast cancer prognosis. Cancer Epidemiol Biomarkers Prev Publ Am Assoc Cancer Res Am Soc Prev Oncol. 2011;20(5):854-8.

31. Fink BN, Steck SE, Wolff MS, Britton JA, Kabat GC, Gaudet MM, et al. Dietary flavonoid intake and breast cancer survival among women on Long Island. Cancer Epidemiol biomarkers Prev Publ Am Assoc Cancer Res Am Soc Prev Oncol. 2007;16(11):2285-92.

32. Guha N, Kwan ML, Quesenberry Jr CP, Weltzien EK, Castillo AL, Caan BJ. Soy isoflavones and risk of cancer recurrence in a cohort of breast cancer survivors: the Life After Cancer Epidemiology study. Breast Cancer Res Treat. 2009;118(2):395-405.

33. Nechuta SJ, Caan BJ, Chen WY, Lu W, Chen Z, Kwan ML, et al. Soy food intake after diagnosis of breast cancer and survival: an indepth analysis of combined evidence from cohort studies of US and Chinese women. Am J Clin Nutr. 2012;96(1):123-32.

34. Shu XO, Zheng Y, Cai H, Gu K, Chen Z, Zheng W, et al. Soy food intake and breast cancer survival. Jama. 2009;302(22):2437-43.

35. Taylor CK, Levy RM, Elliott JC, Burnett BP. The effect of genistein aglycone on cancer and cancer risk: a review of in vitro, preclinical, and clinical studies. Nutr Rev. 2009;67(7):398-415.

36. Lamartiniere CA, Moore JB, Brown NM, Thompson R, Hardin MJ, Barnes S. Genistein suppresses mammary cancer in rats. Carcinogenesis. 1995;16(11):2833-40.

37. Vantyghem SA, Wilson SM, Postenka CO, Al-Katib W, Tuck AB, Chambers AF. Dietary genistein reduces metastasis in a postsurgical orthotopic breast cancer model. Cancer Res. 2005;65(8):3396-403.

38. Jacobs ET, Thomson CA, Flatt SW, Al-Delaimy WK, Hibler EA, Jones LA, et al. Vitamin D and breast cancer recurrence in the Women's Healthy Eating and Living (WHEL) Study. Am J Clin Nutr. 2011;93(1):108-17.
39. Clark AS, Chen J, Kapoor S, Friedman C, Mies C, Esserman L, et al. Pretreatment vitamin D level and response to neoadjuvant chemotherapy in women with breast cancer on the I-SPY trial (CALGB 150007/150015/ACRIN6657). Cancer Med. 2014;3(3):693-701.

40. Lohmann AE, Chapman JA, Burnell MJ, Levine MN, Tsvetkova E, Pritchard KI, et al. Prognostic associations of 25 hydroxy vitamin D in NCIC CTG MA.21, a phase III adjuvant randomized clinical trial of three chemotherapy regimens in high-risk breast cancer. Breast Cancer Res Treat. 2015;150(3):605-11. This observational study nested in a phase III adjuvant trial examined the association between vitamin $D$ levels and measures of recurrence and survival in breast cancer survivors. They reported no association between vitamin $D$ level and any outcome.

41. Pritchard KI, Shepherd LE, Chapman JA, Norris BD, Cantin J, Goss PE, et al. Randomized trial of tamoxifen versus combined tamoxifen and octreotide LAR Therapy in the adjuvant treatment of early-stage breast cancer in postmenopausal women: NCIC CTG MA.14. J Clin Oncol Off J Am Soc Clin Oncol. 2011;29(29):3869-76.

42. Krishnan AV, Swami S, Feldman D. Vitamin D and breast cancer: inhibition of estrogen synthesis and signaling. J Steroid Biochem Mol Biol. 2010;121(1-2):343-8.

43. Goodwin PJ, Ennis M, Pritchard KI, Koo J, Hood N. Prognostic effects of 25-hydroxyvitamin D levels in early breast cancer. Journal of clinical oncology : official journal of the American Society of Clinical Oncology. 2009;27(23):3757-63.

44. Vrieling A, Hein R, Abbas S, Schneeweiss A, Flesch-Janys D, Chang-Claude J. Serum 25-hydroxyvitamin D and postmenopausal breast cancer survival: a prospective patient cohort study. Breast cancer research : BCR. 2011;13(4):R74.

45. Artinian NT, Fletcher GF, Mozaffarian D, Kris-Etherton P, Van Horn L, Lichtenstein AH, et al. Interventions to promote physical activity and dietary lifestyle changes for cardiovascular risk factor reduction in adults a scientific statement from the American Heart Association. Circulation. 2010;122(4):406-41.

46. Arnold M, Leitzmann M, Freisling H, Bray F, Romieu I, Renehan A, et al. Obesity and cancer: an update of the global impact. Cancer Epidemiol. 2016;41:8-15.

47. Noguchi JL, Liss MA, Parsons JK: Obesity, Physical Activity and Bladder Cancer. Curr Urol Rep 2015, 16(10).

48. Hartman SJ, Marinac C, Natarajan L, Patterson R. Obesity, physical activity, and sleep may impact cognitive functioning in breast cancer survivors. Ann Behav Med. 2014;47:S237.

49. Lee JK, So KA, Piyathilake CJ, Kim MK: Mild Obesity, Physical Activity, Calorie Intake, and the Risks of Cervical Intraepithelial Neoplasia and Cervical Cancer. PloS one 2013, 8(6).

50. Martin E, Bulsara C, Battaglini C, Hands B, Naumann FL. Breast and prostate cancer survivor responses to group exercise and supportive group psychotherapy. J Psychosoc Oncol. 2015;33(6):620-34.

51. Falzon C, Radel R, Cantor A, d'Arripe-Longueville F. Understanding narrative effects in physical activity promotion: the influence of breast cancer survivor testimony on exercise beliefs, self-efficacy, and intention in breast cancer patients. Support Care Cancer. 2015;23(3):761-8.

52. Klika RJ, Callahan KE, Golik KS. Exercise capacity of a breast cancer survivor: a case study. Med Sci Sports Exerc. 2008;40(10): $1711-6$.

53. Loprinzi PD, Cardinal BJ, Winters-Stone K, Smit E, Loprinzi CL. Physical activity and the risk of breast cancer recurrence: a literature review. Oncol Nurs Forum. 2012;39(3):269-74.

54. Lahart IM, Metsios GS, Nevill AM, Carmichael AR. Physical activity, risk of death and recurrence in breast cancer survivors: a systematic review and meta-analysis of epidemiological studies. Acta Oncol. 2015;54(5):635-54. This systematic review and meta-analysis is the most recent and thorough evaluation of physical activity and its effects on breast cancer outcomes, including 22 prospective studies. 
55. Holmes MD, Chen WY, Feskanich D, Kroenke CH, Colditz GA. Physical activity and survival after breast cancer diagnosis. Jama-J Am Med Assoc. 2005;293(20):2479-86.

56.• Courneya KS, Segal RJ, McKenzie DC, Dong H, Gelmon K, Friedenreich CM, et al. Effects of exercise during adjuvant chemotherapy on breast cancer outcomes. Med Sci Sports Exerc. 2014;46(9):1744-51. This multicenter RCT is the first trial to examine the effects of exercise on breast cancer outcomes and further demonstrated that exercise may improve these outcomes.

57. Dieli-Conwright CM, Mortimer JE, Schroeder ET, Courneya K, Demark-Wahnefried W, Buchanan TA, et al. Randomized controlled trial to evaluate the effects of combined progressive exercise on metabolic syndrome in breast cancer survivors: rationale, design, and methods. BMC Cancer. 2014;14:238.

58. McTiernan A. Mechanisms linking physical activity with cancer. Nat Rev Cancer. 2008;8(3):205-11.

59. Cuff DJ, Meneilly GS, Martin A, Ignaszewski A, Tildesley HD, Frohlich JJ. Effective exercise modality to reduce insulin resistance in women with type 2 diabetes. Diabetes Care. 2003;26(11):2977-82.

60. Rock CL, Pande C, Flatt SW, Ying C, Pakiz B, Parker BA, et al. Favorable changes in serum estrogens and other biologic factors after weight loss in breast cancer survivors who are overweight or obese. Clin Breast Cancer. 2013;13(3):188-95.

61. Fairey AS, Courneya KS, Field CJ, Bell GJ, Jones LW, Mackey JR. Effects of exercise training on fasting insulin, insulin resistance, insulin-like growth factors, and insulin-like growth factor binding proteins in postmenopausal breast cancer survivors a randomized controlled trial. Cancer Epidemiol Biomark. 2003;12(8):721-7.

62. Irwin ML, Varma K, Alvarez-Reeves M, Cadmus L, Wiley A, Chung GG, et al. Randomized controlled trial of aerobic exercise on insulin and insulin-like growth factors in breast cancer survivors: the Yale Exercise and Survivorship study. Cancer Epidemiol Biomark. 2009;18(1):306-13.

63. Ligibel JA, Campbell N, Partridge A, Chen WY, Salinardi T, Chen $\mathrm{H}$, et al. Impact of a mixed strength and endurance exercise intervention on insulin levels in breast cancer survivors. J Clin Oncol. 2008;26(6):907-12.

64. Schmitz KH, Ahmed RL, Hannan PJ, Yee D. Safety and efficacy of weight training in recent breast cancer survivors to alter body composition, insulin, and insulin-like growth factor axis proteins. Cancer Epidemiol Biomark. 2005;14(7):1672-80

65. Macciò A, Madeddu C, Gramignano G, Mulas C, Floris C, Massa $\mathrm{D}$, et al. Correlation of body mass index and leptin with tumor size and stage of disease in hormone-dependent postmenopausal breast cancer: preliminary results and therapeutic implications. J Mol Med. 2010;88(7):677-86.

66.• Schmidt S, Monk J, Robinson L, Mourtzakis M. The integrative role of leptin, oestrogen and the insulin family in obesity-associated breast cancer: potential effects of exercise. Obes Rev. 2015;16(6):
473-87. This recent review discusses plausible mechanisms underlying the relationship between leptin and obesity, and how energy expenditure through exercise can mediate their interaction.

67. Rogers LQ, Fogleman A, Trammell R, Hopkins-Price P, Vicari S, Rao K, Edson B, Verhulst S, Courneya KS, Hoelzer K: Effects of a physical activity behavior change intervention on inflammation and related health outcomes in breast cancer survivors: pilot randomized trial. Integrative cancer therapies 2012:1534735412449687

68. Schmitz KH, Courneya KS, Matthews C, Demark-Wahnefried W, Galvao DA, Pinto BM, et al. American College of Sports Medicine roundtable on exercise guidelines for cancer survivors. Med Sci Sports Exerc. 2010;42(7):1409-26.

69. Kushi LH, Doyle C, McCullough M, Rock CL, DemarkWahnefried W, Bandera EV, et al. American cancer society guidelines on nutrition and physical activity for cancer prevention reducing the risk of cancer with healthy food choices and physical activity. Ca-Cancer J Clin. 2012;62(1):30-67.

70. Committee PAGA. Physical activity guidelines advisory committee report. Washington, DC: US Department of Health and Human Services; 2008.

71.• Rock CL, Flatt SW, Byers TE, Colditz GA, Demark-Wahnefried W, Ganz PA, et al. Results of the exercise and nutrition to enhance recovery and good health for you (ENERGY) trial: a behavioral weight loss intervention in overweight or obese breast cancer survivors. J Clin Oncol Off J Am Soc Clin Oncol. 2015;33(28):316976. The ENERGY RCT is the largest weight loss intervention trial among breast cancer survivors and demonstrated that a behavioral weight loss intervention can be effective at reducing body weight and circulating levels of estrogens and cytokines implicated in recurrence.

72. Scott E, Daley AJ, Doll H, Woodroofe N, Coleman RE, Mutrie N, et al. Effects of an exercise and hypocaloric healthy eating program on biomarkers associated with long-term prognosis after early-stage breast cancer: a randomized controlled trial. Cancer Causes Control : CCC. 2013;24(1):181-91.

73. Villarini A, Pasanisi P, Traina A, Mano MP, Bonanni B, Panico S, et al. Lifestyle and breast cancer recurrences: the DIANA-5 trial. Tumori. 2012;98(1):1-18.

74. Pierce JP, Stefanick ML, Flatt SW, Natarajan L, Sternfeld B, Madlensky L, et al. Greater survival after breast cancer in physically active women with high vegetable-fruit intake regardless of obesity. J Clinical Oncol Off J Am Soc Clin Oncol. 2007;25(17):2345-51.

75. Chlebowski RT, Aiello E, McTiernan A. Weight loss in breast cancer patient management. J Clin Oncol Off J Am Socc Clin Oncol. 2002;20(4):1128-43.

76. Majed B, Moreau T, Senouci K, Salmon RJ, Fourquet A, Asselain B. Is obesity an independent prognosis factor in woman breast cancer? Breast Cancer Res Treat. 2008;111(2):329-42. 